MANCHESTER

1824

Economics

Discussion Paper Series

EDP-1329

International Environmental Agreements with Uncertainty, Learning and Risk Aversion

Michael Finus

Alistair Ulph

December 2013

Economics

School of Social Sciences

The University of Manchester

Manchester M13 9PL 


\title{
International Environmental Agreements with Uncertainty, Learning and Risk Aversion
}

\author{
Michael Finus \\ (University of Bath) \\ Pedro Pintassilgo \\ (University of Algarve) \\ and \\ Alistair Ulph \\ (University of Manchester)
}

December, 2013

\begin{abstract}
This paper analyses the formation of international environmental agreements (IEAs) under uncertainty, focusing on the role of learning and risk aversion. It bridges two strands of literature: one focused on the role learning for the success of IEA formation when countries are risk neutral and another that explores the implications of uncertainty and risk aversion on IEA formation under no learning. Combining learning and risk aversion seems appropriate as the uncertainties surrounding many international environmental problems are still large, those uncertainties are often highly correlated as for instance in climate change and hence pooling risks may be limited, but those uncertainties are gradually reduced over time through learning. It is shown that the negative conclusion with respect to the role of learning derived for risk neutrality has to be qualified: below a threshold level of risk aversion learning can impact positively on the success of IEAs, above the threshold the opposite is true. Moreover, in a world without full learning (i.e. partial and no learning), risk aversion can lead to better outcomes, but only if risk aversion is sufficiently high.
\end{abstract}

JEL-Classification: C72, D62, D80, Q54

Keywords: international environmental agreements, uncertainty, learning and risk aversion, game theory

We are grateful to Larry Karp for helpful comments on an earlier version of this paper. The usual disclaimer applies. 


\section{Introduction}

Environmental issues such as climate change pose four key challenges for economic analysis: (i) the process of climate change is effectively irreversible; (ii) there are considerable uncertainties about the likely future costs of abatement, but in particular environmental damages; (iii) our understanding of these uncertainties changes over time as a result of learning more about climate science, possible technological responses and behavioral responses by households, firms and governments; (iv) the problem is global, but since there is no single global agency to tackle climate change, policies need to be negotiated through international environmental agreements (IEAs).

The first three issues have been studied quite extensively in the context of a single global government, especially whether the possibility of future learning in a problem with uncertainty and irreversibility leads to more or less current abatement. ${ }^{1}$ The precautionary principle argues for more current abatement, but the theoretical and empirical analysis is more ambiguous. There has also been an extensive literature on the fourth issue, both theoretical and empirical, but mainly in the context of certainty about the net benefits of tackling climate change. ${ }^{2}$ The conclusions have been rather pessimistic, in the sense that while there are substantial benefits to all countries collaborating to tackle climate change, relative to countries acting non-cooperatively, if countries decide independently whether to join an IEA, the relative gains from a stable agreement are small.

More recently, these two strands of literature have begun to be integrated. Ulph and Ulph (1996) and Ulph and Maddison (1997) compare the fully cooperative and the non-cooperative scenarios when countries face uncertainty about damage costs. They show that the value of learning about damage costs may be negative when countries act non-cooperatively and damage costs are correlated across countries. Na and Shin (1998), Ulph (2004), Kolstad (2007), Kolstad and Ulph $(2008,2011)$ have considered how the prospect of future resolution of uncertainty affects the incentives for countries to join an IEA. Again, the results have been

$1 \quad$ See, e.g. Arrow and Fisher (1974), Epstein (1980), Kolstad (1996a,b), Ulph and Ulph (1997), Gollier, Julien and Treich (2000) as well as Narain, Fisher and Hanemann (2007).

2 Classic papers are Carraro and Siniscalco (1993) and Barrett (1994), while for instance Finus (2001, 2003) and Barrett (2003) provide surveys of the literature. 
rather pessimistic: removing the "veil of uncertainty" seems to be detrimental for the success of international environmental cooperation.

All these models have assumed that countries are risk neutral. However, in the climate context, risks are highly correlated and hence possibilities for risk sharing are limited so that the assumption of risk aversion may be quite relevant. Therefore, we extend the two-stage coalition formation setting by Kolstad and Ulph (2008) by departing from the assumption of risk neutrality. They consider a model where countries face common uncertainty about the level of environmental damage costs. ${ }^{3}$ Three scenarios of learning are considered: with full learning, uncertainty about damage costs is resolved before countries decide whether or not to join an IEA; with partial learning, uncertainty is resolved after countries decide whether or not to join an IEA, but before they choose their emissions levels; with no learning, uncertainty is not resolved until countries have decided whether or not to join an IEA and set their emission levels. Kolstad and Ulph (2008) show that the prospect of learning, either full or partial, generally reduces the expected welfare in stable IEAs.

In terms of risk aversion, our work is closely related to three papers to which we add in particular the role of learning. Endres and Ohl (2003) show in a simple two-player prisoners' dilemma, using the mean-standard deviation approach to capture risk aversion, that risk aversion can increase the prospects of cooperation once it reaches a certain threshold. The reason is that the benefits of mutual cooperation increase relative to the payoffs of unilateral cooperation and no cooperation because cooperation reduces the variance of payoffs. The more risk averse players are, the more attractive cooperation becomes compared to freeriding. In their model, there is a first threshold above which the prisoners' dilemma turns into a chicken game and a second threshold above which the game turns into an assurance game. Compared to their paper, we allow for an arbitrary number of players, model cooperation as a two-stage coalition formation game and consider explicitly the role of learning.

\footnotetext{
By common uncertainty we mean that each country faces the same ex-ante distribution of possible damage costs, and when uncertainty is fully resolved they face the same ex-post level of damage costs, i.e. the risks they face are fully correlated across countries. Kolstad and Ulph (2011) extend this model to consider the case where the risks each country faces are uncorrelated. Uncorrelated uncertainty is also considered in a slightly different model in Finus and Pintassilgo (2013) and empirically investigated in a climate model with twelve world regions in Dellink and Finus (2012).
} 
Bramoullé and Treich (2009) consider risk averse players in a global emission model, in which all players behave non-cooperatively as singletons. They show that equilibrium emissions are lower under uncertainty than under certainty, as part of a hedging strategy, but the effect on global welfare is ambiguous. The authors also find that emissions decrease with the level of risk aversion. Unlike our paper, Bramoullé and Treich are not concerned with learning and coalition formation.

Boucher and Bramoullé (2010) consider the effects of risk aversion on coalition formation, but with no learning. They analyze the formation of an international environmental treaty using a similar coalition game and payoff function as adopted in this paper. Using an expected utility approach, their analysis focuses on the effect of uncertainty and risk aversion on signatories' efforts, the participation level in an agreement and total welfare. They show that if additional abatement reduces the variance of countries' payoffs, then, under risk aversion, an increase in uncertainty tends to increase abatement levels and may decrease equilibrium IEA membership while the reverse is true if additional abatement increases the variance of countries' payoffs. ${ }^{4}$ In this paper, our model of no learning satisfies the first condition.

Thus, taken together, in our paper, we generalize the analysis of Kolstad and Ulph (2008) by allowing for risk aversion, and the analysis of Boucher and Bramoulle (2010) and Endres and Ohl (2003) by considering the role of learning. To make the analysis tractable, like Endres and Ohl, we adopt the mean-standard deviation (MS) approach to represent preferences under uncertainty. This was introduced by Markowitz (1952) and Tobin (1958), and is a widely used alternative to the expected utility (EU) criterion for decision-making under uncertainty, introduced by von Neumann and Morgenstern (1944). There have been a large number of studies comparing the relative advantages of each approach, asking under which conditions they are consistent - i.e. they produce the same results. In a path-breaking article Meyers (1987) showed that under a given parameter restriction (LS - location and scale) the two approaches are consistent. This holds in particular if the payoff function is linear in the

$4 \quad$ Boucher and Bramoulle (2010) argue that the effects of uncertainty and risk aversion on the provision of a public good depend on whether one models the situation as provision of a public good or amelioration of a public bad. Hong and Karp (2013) show that this interpretation is incorrect: it does not matter whether one talks of providing a public good or ameliorating a public bad. What matters is whether players' actions increase or decrease the volatility of payoffs. In our model, like in Endres and Ohl (2003) and the emission game in Boucher and Bramoulle (2010), abatement (emissions) reduces (increase) the volatility of payoffs. 
uncertain parameter, which is the case in our model (see equation 1 below). Saha (1997) argues that, despite the consistency of the two criteria, the MS is generally more flexible in representing alternative risk preferences and is simpler in terms of empirical applications. One main advantage is that it includes explicitly the two moments of the payoffs, as for a large class of preferences they contain all the relevant information of a decision problem under uncertainty. According to Saha, this explains why the MS criterion is so widely used, both in theoretical and empirical studies.

The paper proceeds as follows. In section 2, we set out the theoretical model and present our results in section 3. Section 4 summarizes our main conclusions.

\section{The Model}

\section{$2.1 \quad$ No Uncertainty}

There are $N$ identical countries, indexed $i=1, \ldots, N$. Each country produces emissions $x_{i}$ with aggregate emissions denoted by $X=\sum_{i=1}^{N} x_{i}$. Aggregate emissions cause global environmental damages. The cost of environmental damages per unit of global emissions is $\gamma$ and the benefit per unit of individual emissions is normalized to 1. (Thus, $\gamma$ essentially measures the cost-benefit ratio.) The payoff to country $i$, as a function of emissions, is given by

$\Pi_{i}\left(x_{i}, X\right) \equiv x_{i}-\gamma X$

In this simple model, the (continuous) strategy space can be normalized to $x_{i} \in[0,1]$. Due to the linearity of the payoff function, players either choose $x_{i}=1$ (full pollution), $x_{i}=0$ (no pollution) or they are indifferent between any level in the range $x_{i} \in[0,1]$ (partial pollution). Following Kolstad (2007) and Kolstad and Ulph (2008) it is assumed that in the case of indifference, players choose $x_{i}=0$. Hence, in the following, we have two possible discrete strategies: pollution or no abatement $\left(x_{i}=1\right)$ and no pollution or abatement $\left(x_{i}=0\right)$.

In order to make this model interesting, we require that the individual benefit exceeds the individual unit damage cost from pollution, i.e. $1>\gamma$ (hence countries pollute in the Nash equilibrium) but does not exceed the global unit damage cost, i.e. $1<\gamma N$ (hence countries abate in the social optimum), which together imply: 


\section{Assumption 1: $\quad 1 / N<\gamma<1$.}

In order to study coalition formation, we employ the widely used two-stage model on IEA formation as introduced by Carraro and Siniscalco (1993) and Barrett (1994), borrowed from the literature on cartel formation (d'Aspremont et al. 1983), which is solved backwards. In the second stage, the emission game, for any arbitrary number of IEA members $n, 1 \leq n \leq N$, the members of the IEA (which we denote by the symbol $c$ for coalition countries) and the remaining countries (which we denote by the symbol $f$ for fringe countries) set their emission levels as the outcome of a Nash game between the coalition and the fringe countries. ${ }^{5}$ That is, the coalition members together maximize the aggregate payoff to their coalition, whereas fringe countries maximize their own payoff. As we assumed $1>\gamma, x_{i}^{f}=1$ follows; coalition members will chose $x_{i}^{c}=0$ provided $1 \leq \gamma n$, and so $\Pi_{i}^{f}(n)=1-\gamma(N-n)$ and $\Pi_{i}^{c}(n)=-\gamma(N-n)$; however if $1>\gamma n$, then coalition members will also pollute, $x_{i}^{c}=1$ and so $\Pi_{i}^{c}(n)=\Pi_{i}^{f}(n)=1-\gamma N^{6}{ }^{6}$

Knowing the payoffs to coalition and fringe countries for any arbitrary number of IEA members, we then determine the equilibrium in the first stage, the membership game, again, as a Nash equilibrium: no coalition member could become better off from leaving the coalition, with $C$ denoting the set of coalition members, and no fringe country could be made better off from joining the coalition: ${ }^{7}$

Internal stability: $\quad \forall i \in C: \Pi_{i}^{c}(n) \geq \Pi_{i}^{f}(n-1)$

External stability: $\quad \forall i \notin C: \Pi_{i}^{f}(n)>\Pi_{i}^{c}(n+1)$.

Consider a coalition with $n$ members that abates because $1 \leq \gamma n$. Now if one member left the coalition and it still paid the remaining $n-1$ to abate, i.e. $1 \leq \gamma(n-1)$, internal stability $\Pi_{i}^{c}(n) \geq \Pi_{i}^{f}(n-1)$ would require $0-\gamma(N-n) \geq 1-\gamma(N-n+1)$, or equivalently $\gamma \geq 1$, which violates Assumption 1. Hence, we require that it does not pay the remaining countries

5 A sequential Stackelberg game in the second stage, as an alternative assumption (e.g. Barrett 1994), would make no difference here as players have dominant strategies. This is also applies to Boucher and Bramoullé (2010).

6 It is now evident why we need Assumption 1: it avoids trivial outcomes where all countries either abate or pollute no matter whether they are coalition members or fringe countries.

7 Without loss of generality, the strong inequality could be replaced by a weak inequality sign for external stability. Our assumption avoids knife-edge cases where a fringe country is indifferent between staying outside and joining a coalition. 
to abate once a member leaves, i.e. $1>\gamma(n-1)$, and then internal stability requires $0-\gamma(N-n) \geq 1-\gamma N$ which implies that $n \geq 1 / \gamma$. Thus, the internally stable coalition $n^{*}$ is the smallest integer $I(\gamma)$ no less than $1 / \gamma$ which, as can be easily checked, is also externally stable and hence stable. It is straightforward to see that $I(\gamma)$ is a non-increasing function of $\gamma^{8}$

Since ex-ante all countries are identical, there is no explicit process for determining which countries are selected as IEA members and which as fringe countries. We shall assume, following Kolstad and Ulph (2008) and Rubio and Ulph (2007) that there is random process for determining which countries become IEA members. Thus, we define the average or expected equilibrium payoff per country by $\bar{\Pi}_{i}^{*} \equiv\left(n^{*} / N\right) \Pi_{i}^{c}\left(n^{*}\right)+\left(\left(N-n^{*}\right) / N\right) \Pi_{i}^{f}\left(n^{*}\right)$, which is a strictly decreasing function of $\gamma .{ }^{9}$

Thus, this simple model provides a relationship between the unit damage cost $\gamma$ and the equilibrium number of coalition members. The equilibrium is a knife-edge equilibrium with $n^{*}$ countries forming the coalition, which de facto dissolves once a member leaves the coalition as no country would abate anymore. The equilibrium coalition size weakly decreases in the cost-benefit ratio from emissions $\gamma$ - the larger $\gamma$ the less countries are needed to make cooperation profitable.

\subsection{Uncertainty}

Now assume that the unit damage cost of global emissions is uncertain and equal for all countries, both ex-ante and ex-post. We denote the value by $\gamma_{s}$ in the state of the world $s$ and hence (1) becomes:

$\Pi_{i, s}\left(x_{i}, X\right) \equiv x_{i}-\gamma_{s} X$

$8 \quad$ Kolstad and Ulph (2008) use the approximation $I(\gamma)=1 / \gamma$ which ignores the integer nature of $I(\gamma)$. Then $I(\gamma)$ can be considered a strictly decreasing and convex function of $\gamma$. However, as Karp (2012) pointed out, the original function $I(\gamma)$ is neither convex nor concave.

$9 \quad$ Using $\Pi_{i}^{c}$ and $\Pi_{i}^{f}$ from above, noting $n^{*}=I(\gamma)$, then $\bar{\Pi}_{i}^{*}=1-\gamma N+I(\gamma)(\gamma-1 / N)$. Consider an infinitesimal variation $\Delta \gamma>0$, such that $I(\gamma)$ does not change. Then, $\Delta \bar{\Pi}_{i}^{*}=(-N+I(\gamma)) \Delta \gamma<0$, $\forall I(\gamma)<N$. However, if $\Delta \gamma>0$ implies $\Delta I(\gamma)<0$, then $\Delta \bar{\Pi}_{i}^{*}=(-N+I(\gamma)) \Delta \gamma$ $+\Delta I(\gamma)(\gamma-1 / N)<0$, which completes the proof. Using the approximation in footnote 8 (ignoring the integer nature of $I(\gamma)$ ), then $\bar{\Pi}_{i}^{*}$ is a strictly decreasing concave function of $\gamma$. See Kolstad and Ulph (2008). 
For simplicity, we assume that $\gamma_{s}$ can take one of two values: low damage costs, $\gamma_{l}$, with probability $p$, and high damage costs, $\gamma_{h}$ with probability $(1-p)$, where $\gamma_{l}<\gamma_{h}$ and $0<p<1$. We denote by $\bar{\gamma} \equiv p \gamma_{l}+(1-p) \gamma_{h}$ the expected value of unit damage costs, and by $\sigma\left(\gamma_{s}\right) \equiv\left(\gamma_{h}-\gamma_{l}\right) \sqrt{p(1-p)}$ the standard deviation of unit damage costs.

To assess how countries evaluate payoffs across states of the world, we assume that each country's attitude to risk can be represented by a mean-standard deviation (MS) utility function, which is the same for all countries:

$$
V_{i}\left(x_{i}, X\right) \equiv E\left[\Pi_{i}\left(x_{i}, X\right)\right]-\alpha \sigma\left[\Pi_{i}\left(x_{i}, X\right)\right]
$$

where $\alpha \geq 0$ is the coefficient of risk aversion ${ }^{10}$; i.e. the utility to a country is the expected payoff minus the standard deviation of payoffs weighted by the factor $\alpha$. $\alpha=0$ corresponds to risk neutrality.

For later purposes, it will be useful to define:

$\hat{\gamma} \equiv \bar{\gamma}+\alpha \sigma\left(\gamma_{s}\right) \geq \bar{\gamma}$

as the "risk-adjusted expected unit damage cost".

While ex-ante countries face uncertainty about the true value of unit damage costs, we want to allow for the possibility that countries may learn information during the course of the game which changes the risk they face. We shall follow Kolstad and Ulph (2008) in considering three very simple scenarios of learning. With No Learning (NL) countries make their decisions about membership and emissions with uncertainty about the true value of unit damage costs. With Full Learning (FL) countries learn the true value of unit damage costs before they have to take their decisions on membership and emissions. With Partial Learning (PL) countries learn the true value of damage costs after they have made their membership decisions but before they make their emission decisions. Thus, in this simple analysis, learning takes the form of revealing perfect information.

As in the model without uncertainty, we have to introduce some parameter restrictions. Moreover, the equilibrium size of the coalition can be related to unit damage costs. We define

10 We could consider as in Endres and Ohl (2003) that $\alpha$ can be negative if players are risk loving. However, we discard this possibility in order to keep the discussion as brief as possible. Note that from (5) it is apparent that emissions (abatement) increase (decrease) the volatility of payoffs. See footnote 4. 
$n_{h} \equiv I\left(\gamma_{h}\right), n_{l} \equiv I\left(\gamma_{l}\right), \quad \bar{n} \equiv I(\bar{\gamma}), \quad \hat{n} \equiv I(\hat{\gamma})$ and $n_{N}=N$. It will turn out that stable IEAs will take one of these values. For sensible results, we make the following assumption.

Assumption 2: $\quad$ (i) $1 / N<\gamma_{l}<\bar{\gamma}<\gamma_{h}<1$

(ii) $\quad n_{h}<\bar{n}<n_{l} \leq n_{N}$.

Assumption 2(i) is essentially Assumption 1 in the context of uncertainty and risk aversion. Assumption 2(ii) basically states that there are differences (at least 1) between the sizes of the stable IEAs under uncertainty. For the theoretical analysis, it is helpful to consider two parameter constellations, which we shall call case 1 and 2 . The two cases are related to the level of risk aversion, with $\alpha^{T}$ a threshold value, noting that the value of risk aversion $\alpha$ affects the level of the risk-adjusted expected unit damage cost $\hat{\gamma}$.

Cases: $\quad$ (i) $\quad$ Case 1: $\hat{\gamma}<1 \Rightarrow 0 \leq \alpha<(1-\bar{\gamma}) / \sigma\left(\gamma_{s}\right) \equiv \alpha^{T}$ and

(ii) Case 2: $\hat{\gamma} \geq 1 \Rightarrow \alpha \geq(1-\bar{\gamma}) / \sigma\left(\gamma_{s}\right) \equiv \alpha^{T}$.

Case 1 is essentially Assumption 1 in the context of uncertainty and risk aversion; abatement never pays for a single country. However, now we want to allow with case 2 for the possibility that if players are sufficiently risk averse, then even a single player may decide to abate. Note that case 1 includes the case of risk neutrality $(\alpha=0)$. It will turn out that going from case 1 to case 2 leads to a regime shift. From a statistical point of view, it is clear that as long as the degree of risk aversion is sufficiently low, the restriction $\hat{\gamma}<1$ is not really binding. Only for large degrees of risk aversion will the likelihood of $\hat{\gamma} \geq 1$ become significant and, for large enough levels of $\alpha$, the likelihood of $\hat{\gamma} \geq 1$ may even exceed that of $\hat{\gamma}<1$.

Note that now with uncertainty the expected utility per country from an ex-ante perspective is $\bar{V}_{i} \equiv(n / N) \bar{V}_{i}^{c}(n)+((N-n) / N) \bar{V}_{i}^{f}(n)$ where in equilibrium $n$ may take on one of the values mentioned above (i.e. $n_{h}, n_{l}, \bar{n}, \hat{n}$ or $n_{N}$ ).

\section{Results}

\subsection{Analytical Results}

In this sub-section, we generalize the results of Kolstad and Ulph (2008) who assume risk neutrality ( $\alpha=0)$. In terms of the risk aversion parameter $\alpha$, we distinguish between case 1 
and case 2 as spelled out in sub-section 2.2 above. We start with the benchmark scenario of Full Learning (FL). Players know the realization of the damage parameter $\gamma$ at the outset of the coalition formation game. Thus, results follow directly from what we know from a game with certainty and hence risk aversion does not play a role. However, it has to be pointed out that - even for FL - an evaluation has to take an ex-ante perspective in order to allow for a sensible comparison of coalition sizes and utility levels, across different scenarios of learning, for a given level of risk aversion.

\section{Proposition 1: Full Learning}

If state $s=l, h$ has been revealed at the outset, then in the emission game, fringe members always pollute, and coalition members abate if $n \geq n_{s}=I\left(\gamma_{s}\right)$. In the membership game, the stable IEA has $n_{s}$ members; the utility to a coalition member is $V_{i, F L}^{c}(s)=-\gamma_{s}\left(N-n_{s}\right)$ and to a fringe country it is $V_{i, F L}^{f}(s)=1-\gamma_{s}\left(N-n_{s}\right)$. Thus, in state $s$, the expected utility per country is $\bar{V}_{i, F L}(s)=\left(N-n_{s}\right)\left(1 / N-\gamma_{s}\right)$. From an ex-ante perspective, the expected size of an IEA is $\bar{n}_{F L}=p n_{l}+(1-p) n_{h}$ and the expected utility per country is:

$$
\begin{array}{r}
\bar{V}_{i, F L}=p \bar{V}_{i, F L}(s=l)+(1-p) \bar{V}_{i, F L}(s=h)=(1-N \bar{\gamma})+p n_{l}\left(\gamma_{l}-1 / N\right) \\
+(1-p) n_{h}\left(\gamma_{h}-1 / N\right) .
\end{array}
$$

Hence, risk aversion neither affects the coalition size nor expected utility.

Proof: Is identical to the case of risk neutrality in Kolstad and Ulph (2008). Q.E.D.

For FL, the outcome in each state $s$ in terms of the size of a stable IEA, the utility to coalition and fringe countries and hence the expected utility per country is just the same as in an IEA game where the level of damage costs $\gamma_{s}$ is known with certainty. Thus, by taking expectations across the two states of the world, we obtain the expected size of an IEA and the expected utility per country.

\section{Proposition 2: No Learning}

Case 1: $\hat{\gamma}<1\left(0 \leq \alpha<(1-\bar{\gamma}) / \sigma\left(\gamma_{s}\right) \equiv \alpha^{T}\right)$

In the emission game, fringe countries always pollute, while coalition members abate if $n \geq \hat{n}$ and pollute otherwise. In the membership game, the unique stable IEA has $n_{N L}=\hat{n}=I(\hat{\gamma})$ members abating and $(N-\hat{n})$ fringe countries polluting, with $\hat{n} \leq \bar{n}$. The expected utility to a 
coalition member is $\bar{V}_{i, N L}^{c}=-\hat{\gamma}(N-\hat{n})$ and to a fringe country it is $\bar{V}_{i, N L}^{f}=1-\hat{\gamma}(N-\hat{n})$ and hence expected utility per country is $\bar{V}_{i, N L}=(N-\hat{n})(1 / N-\hat{\gamma})$.

The size of the stable coalition $n_{N L}$ is weakly decreasing and the expected utility per country is strictly decreasing in the risk aversion parameter $\alpha$.

Case 2: $\hat{\gamma} \geq 1\left(\alpha \geq(1-\bar{\gamma}) / \sigma\left(\gamma_{s}\right) \equiv \alpha^{T}\right)$

In the emission game, all players abate and hence $n_{N L} \in\{1, \ldots, N\}$ and $\bar{V}_{i, N L}=0$ for all levels of risk aversion above threshold $\alpha^{T}$.

Proof: Case 1: Follows the same steps as in Kolstad and Ulph (2008) by replacing $n_{N L}=\bar{n}=I(\bar{\gamma})$ by $n_{N L}=\hat{n}=I(\hat{\gamma})$. Case 2: noting that for $\hat{\gamma} \geq 1$ the dominant strategy of both coalition members and fringe countries is to abate, for any coalition size, the result is immediate. Q.E.D.

With respect to case 1 , for $\alpha=0$, the result displays certainty equivalence in the sense that the outcome is the same as would be obtained if countries faced unit damage costs $\bar{\gamma}$ with certainty and no risk. Any mean-preserving increase in risk related to the uncertain damage cost parameter has no effect on either the expected coalition size or expected welfare under risk neutrality. This is different with risk aversion, i.e. $\alpha>0$ (though $\alpha<\alpha^{T}$ ): perceived (risk-adjusted) unit damage cost increase with the degree of risk aversion and since the size of the stable coalition decreases in (risk-adjusted) unit damage costs, risk aversion leads to smaller equilibrium coalitions. Note that for No Learning (NL), and different from FL, coalition size and expected coalition size are the same.

The impact of risk aversion on expected per country utility is negative due to two effects. ${ }^{11}$ First for a given coalition, by assumption, expected utility of coalition members and fringe countries decreases in risk aversion, which is the direct effect. Second, expected utility per country decreases in the coalition size and - as just pointed out - risk aversion lowers the equilibrium coalition size, which may be viewed as an indirect effect.

11 Note that strictly speaking utility cannot be compared for different degrees of risk aversion. Therefore, this result will only be used later when comparing utility across different scenarios of learning for the same degree of risk aversion. 
With respect to case 2, if the degree of risk aversion passes a threshold $\alpha^{T}$ such that the riskadjusted unit damage cost parameter exceeds $1, \hat{\gamma} \geq 1$, even for fringe players abatement pays. This may be interpreted as all coalitions being stable or that there is no need for cooperation as even in the non-cooperative equilibrium all players abate.

Taken together, risk aversion has a negative impact on coalition size and expected per country utility, but once a threshold is reached, this leads to a regime shift with full cooperation.

\section{Proposition 3: Partial Learning}

Case 1: $\hat{\gamma}<1\left(0 \leq \alpha<(1-\bar{\gamma}) / \sigma\left(\gamma_{s}\right) \equiv \alpha^{T}\right)$

(i) There are two possible stable IEAs: $n_{P L}^{l}=n_{h}<n_{l}=n_{P L}^{2}$. In the first possible stable IEA of size $n_{P L}^{l}=n_{h}$, coalition members pollute in the low damage cost state and abate in the high damage cost state and fringe countries pollute in both states. In the second possible stable IEA of size $n_{P L}^{2}=n_{l}$, coalition members abate in both states of the world and fringe countries pollute in both states of the world.

(ii) There always exists a stable IEA, either $n_{h}$ or $n_{l}$, or both.

(iii) For $\alpha=0, n_{P L}^{l}=n_{h}$ is always a stable IEA whereas $n_{P L}^{2}=n_{l}$ is a stable IEA if and only if $p \geq p^{T}$ where $p^{T} \equiv\left(1-\gamma_{h}\right) /\left(n_{l} \gamma_{l}-\gamma_{h}\right)$. If $n_{P L}^{2}=n_{l}$ is stable, it Pareto-dominates $n_{P L}^{1}=n_{h}$.

(iv) For $0<\alpha<\alpha^{T}, n_{h}$ will be a stable IEA if

either: (a) $\quad N \geq \breve{N}$ where $\breve{N} \equiv \frac{n_{h} \gamma_{h}-\frac{1}{2}\left(1-\gamma_{h}\right)}{\left(\gamma_{h}-\gamma_{l}\right)}$

or: $\quad$ (b) $\quad N<\breve{N}$ and $0<\alpha \leq \breve{\alpha} \equiv \frac{(1-p)\left(1-\gamma_{h}\right)}{\sqrt{p(1-p)}\left(\gamma_{h}-\gamma_{l}\right)(2(\breve{N}-N))}$.

The expected utility to coalition and fringe countries is given by:

$$
\begin{aligned}
& \bar{V}_{i, P L}^{c}\left(n_{h}\right)=-\hat{\gamma} N+p+(1-p) \gamma_{h} n_{h}-\alpha\left(1-\gamma_{h} n_{h}\right) \sqrt{p(1-p)} \text { and } \\
& \bar{V}_{i, P L}^{f}\left(n_{h}\right)=1-\bar{\gamma} N+(1-p) \gamma_{h} n_{h}-\alpha \sqrt{p(1-p)\left(N\left(\gamma_{h}-\gamma_{l}\right)-n_{h} \gamma_{h}\right)^{2}} .
\end{aligned}
$$

The expected payoff per country is given by: 
$\bar{V}_{i, P L}\left(n_{h}\right)=1-\bar{\gamma} N+(1-p) n_{h}\left(\gamma_{h}-1 / N\right)-\alpha \sqrt{p(1-p)}\left(\frac{n_{h}}{N}\left(1+A-\sqrt{A^{2}}\right)+\sqrt{A^{2}}\right)$ where $A \equiv N\left(\gamma_{h}-\gamma_{l}\right)-n_{h} \gamma_{h}$.

(v) For $0<\alpha<\alpha^{T}, n_{l}$ will be a stable IEA if:

(a) $N \geq \tilde{N}, p>p^{T}$ and $0<\alpha \leq \tilde{\alpha}$ where $\tilde{N} \equiv \frac{\gamma_{h}\left(n_{l}-1\right)}{\left(\gamma_{h}-\gamma_{l}\right)}$ and $\tilde{\alpha} \equiv \frac{\left(p-p^{T}\right)}{\sqrt{p(1-p)}}$;

(b) $\tilde{\tilde{N}}<N<\tilde{N}, \quad p>p^{T}$ and $0<\alpha \leq \tilde{\tilde{\alpha}}$ where $\tilde{\tilde{N}} \equiv \frac{n_{l}\left(\gamma_{h}-\gamma_{l}\right)+\gamma_{h}\left(n_{l}-1\right)}{2\left(\gamma_{h}-\gamma_{l}\right)}<\tilde{N}$ and $\tilde{\tilde{\alpha}} \equiv \frac{\left(p-p^{T}\right)\left(n_{l} \gamma_{l}-\gamma_{h}\right)}{2 \sqrt{p(1-p)}\left(\gamma_{h}-\gamma_{l}\right)(N-\tilde{\tilde{N}})}$;

(c) $N \leq \tilde{\tilde{N}}$ and $p \geq p^{T}$;

(d) $N \leq \tilde{\tilde{N}}, \quad p<p^{T}$ and $\alpha \geq \tilde{\tilde{\alpha}}$ where $\tilde{\tilde{N}}>\breve{N}$ and $\tilde{\tilde{\alpha}}<\breve{\alpha}$.

The expected utility to coalition and non-coalition countries is given by: $\bar{V}_{i, P L}^{c}\left(n_{l}\right)=-\hat{\gamma}\left(N-n_{l}\right)$ and $\bar{V}_{i, P L}^{f}\left(n_{l}\right)=1-\hat{\gamma}\left(N-n_{l}\right)$, respectively. The expected utility per country is given by $\bar{V}_{i, P L}\left(n_{l}\right)=1-\hat{\gamma}\left(N-n_{l}\right)-\frac{n_{l}}{N}$.

(vi) The expected per country utility for coalition size $n_{P L}^{1}=n_{h}$ strictly decreases with the level of risk aversion.

(vii) The expected per country utility for coalition size $n_{P L}^{2}=n_{l}$ (weakly) decreases with the level of risk aversion, and strictly decreases if $n_{l}<N$.

Case 2: $\hat{\gamma} \geq 1\left(\alpha \geq(1-\bar{\gamma}) / \sigma\left(\gamma_{s}\right) \equiv \alpha^{T}\right)$

(viii) There are two possible stable IEAs: $n_{P L}^{1}=n_{h}$ and $n_{P L}^{2}=N$.

(ix) $\quad n_{P L}^{l}=n_{h}$ is a stable IEA under the same conditions as in (iv) and the implications mentioned in (i).

(x) $\quad n_{P L}^{2}=N$ is always a stable IEA: all countries abate and hence expected utility per country is $\bar{V}_{i, P L}=0$.

(xi) Equilibrium $n_{P L}^{2}=N$ Pareto-dominates $n_{P L}^{l}=n_{h}$.

Proof: See Appendix 1. 
As is clear from the results above, Partial Learning (PL) is the most complex of the three learning scenarios. In the second stage, the emission game, the value of the damage parameter $\gamma$ is revealed to the players. Hence, the emission game is like the game under FL. Importantly, and very different from NL, this implies that fringe players, or singletons, never abate, regardless of the degree of risk aversion ((i) and (ix)). Thus, different from NL, the grand coalition in case 2 comes about only due to the membership game (and not due to the emission game as for NL) where decisions - different from FL - have to be based on expected utility. Since the grand coalition always Pareto-dominates the smaller equilibrium coalition, it is assume that full cooperation is the outcome in case 2 . Thus, in case, 2 , if risk aversion is above the threshold $\alpha^{T}$, the grand coalition forms and hence risk aversion leads to a better outcome than risk neutrality.

In case 1 , if risk aversion is below the threshold $\alpha^{T}$, things are more complex. There are two possible stable IEAs, $n_{h}$ and $n_{l}$ (i), and there always exists a stable IEA, either $n_{h}$ or $n_{l}$ or both (ii). For the benchmark case $\alpha=0$, risk neutrality, $n_{h}$ is always a stable IEA (iii) whereas the coalition with the larger size, $n_{l}$, is only stable if and only if $p \geq p^{T}$ (iii). From the definition of $p^{T}$ it is evident that this condition only holds if the probability of the low damage cost $p$ is close to 1 (a parameter constellation not very interesting when analyzing risk), recalling that $n_{l}$ is the smallest integer no less than $1 / \gamma_{l}$ and hence $n_{l} \gamma_{l}$ and $p^{T}$ tend to be close to 1 . However, Assumption 2 implies that the probability of low damage $p$ cannot be too high or too low. Thus, as argued by Kolstad and Ulph (2008), $n_{l}$ is not likely under risk neutrality. ${ }^{12}$

For risk aversion, results (iv) and (v) can be summarized as follows. For the interesting and most likely case of $p<p^{T}$ if the number of players is large $(N>\tilde{\tilde{N}}>\breve{N})$ only $n_{h}$ is stable. For an intermediate number of players $(\tilde{\tilde{N}} \geq N \geq \breve{N}), n_{h}$ is stable and $n_{l}$ is also stable if the level of risk aversion is sufficiently high, i.e. $\alpha \geq \tilde{\tilde{\alpha}}$. For a small number of players $(\tilde{\tilde{N}}>\breve{N}>N)$, only $n_{h}$ is stable if $\alpha<\tilde{\tilde{\alpha}}$, both $n_{h}$ and $n_{l}$ are stable if $\tilde{\tilde{\alpha}} \leq \alpha \leq \breve{\alpha}$ and only $n_{l}$ is stable if $\alpha>\breve{\alpha}$. Thus, risk aversion makes the second and larger equilibrium $n_{l}$ also possible for $p<p^{T}$. Moreover, an increase in the level of risk aversion makes the smaller equilibrium $n_{h}$ less likely and the larger equilibrium $n_{l}$ more likely, provided that the number

12 In the next section, we show through Monte Carlo simulations that the proportion of admissible parameters for which $p \geq p^{T}$ is in fact quite low. 
of players is not too large. For the less interesting case of $p \geq p^{T}$, also with risk aversion the larger equilibrium emerges under most parameter constellations of $N$ and $\alpha$.

In terms of expected per country utility, Proposition 3 stresses that in case 1, like under NL, the expected per country utility under PL decreases with risk aversion in each equilibrium, $n_{h}$ and $n_{l}$ (vi and vii). ${ }^{13}$ This is the direct negative effect which risk has on expected per country utility. However, the indirect effect tends to go in the opposite direction. An increase in the level of risk aversion makes the large second equilibrium $n_{P L}^{2}=n_{l}$ more likely, with typically higher expected per country utility than the smaller equilibrium $n_{P L}^{l}=n_{h}$. Hence, the overall impact of risk on expected utility cannot be theoretically deduced. The simulation runs on which we report in the next sub-section show that the overall impact is on average negative.

We now compare the outcomes in terms of expected size of the IEA and expected utility per country across the three models of learning. In order to benchmark this with previous results, we consider under case 1 the special case of $\alpha=0$.

\section{Proposition 4: Comparison of Outcomes under the Three Scenarios of Learning}

Case 1: Case 1: $\hat{\gamma}<1\left(0 \leq \alpha<(1-\bar{\gamma}) / \sigma\left(\gamma_{s}\right) \equiv \alpha^{T}\right)$

a) $\alpha=0$

(i) $\quad n_{P L}^{1}=n_{h}<n_{N L}=\bar{n}<n_{P L}^{2}=n_{l}$;

(ii) $\quad n_{P L}^{l}=n_{h}<\bar{n}_{F L}<n_{P L}^{2}=n_{l}$;

(iii) $\quad \bar{n}_{F L} \geq n_{N L}-1$;

(iv) $\quad \bar{V}_{i, P L}\left(n_{h}\right)<\bar{V}_{i, N L}(\bar{n})<\bar{V}_{i, P L}\left(n_{l}\right)$;

(v) $\bar{V}_{i, P L}\left(n_{h}\right)<\bar{V}_{i, F L}<\bar{V}_{i, P L}\left(n_{l}\right)$.

b) $\alpha>0$

\section{$\underline{\text { Coalition Size }}$}

(vi) $n_{P L}^{1}=n_{h}<n_{l}=n_{P L}^{2}$ and $n_{N L}=\hat{n}<n_{l}=n_{P L}^{2}$ always hold. Moreover, there is a threshold of $\alpha, \alpha_{1}$, such that for $\alpha_{1} \leq \alpha \leq \alpha^{T}, n_{l}=n_{P L}^{2}>n_{P L}^{1} \geq \hat{n}=n_{N L}$.

(vii) There is a threshold of $\alpha, \alpha_{2}, \alpha_{2}<\alpha_{1}$, such that for $\alpha_{2} \leq \alpha \leq \alpha^{T}, \bar{n}_{F L} \geq n_{N L}=\hat{n}$.

13 The same qualification as mentioned in footnote 11 applies. 
(viii) In general, $n_{P L}^{1}=n_{h}<\bar{n}_{F L}<n_{l}=n_{P L}^{2}$; for $N<\breve{N}$ and $\breve{\alpha}<\alpha<\alpha^{T}$ (defined in Proposition 3) the unique stable IEA for PL will be $n_{P L}^{2}=n_{l}>\bar{n}_{F L}$.

\section{$\underline{\text { Utility }}$}

(ix) There is a threshold of $\alpha, \alpha_{3}, \alpha_{3}<\alpha_{1}$, such that for $\alpha_{3} \leq \alpha \leq \alpha^{T}, \bar{V}_{i, F L} \geq \bar{V}_{i, N L}(\hat{n})$.

(x) There is a threshold value of $\alpha, \alpha_{4}$ such that for $\alpha_{4} \leq \alpha<\alpha^{T}$ $\bar{V}_{i, F L} \geq \bar{V}_{i, P L}\left(n_{P L}^{2}\right)>\bar{V}_{i, P L}\left(n_{P L}^{1}\right)$. Otherwise, $\bar{V}_{i, P L}\left(n_{P L}^{2}\right) \geq \bar{V}_{i, F L} \geq \bar{V}_{i, P L}\left(n_{P L}^{l}\right)$.

(xi) $\quad \bar{V}_{i, P L}\left(n_{l}\right)>\bar{V}_{i, N L}(\hat{n})$.

Case 2: $\hat{\gamma} \geq 1\left(\alpha \geq(1-\bar{\gamma}) / \sigma\left(\gamma_{s}\right) \equiv \alpha^{T}\right)$

(xii) For parameter values set out in Proposition 3, $n_{h}$ is a stable IEA for PL; its relationship to stable IEAs for FL and NL are as set out in Case 1.

(xiii) For all parameter values the other stable IEA under PL is $n_{P L}^{2}=n_{N}=N$; then $N=n_{P L}^{2}=n_{N L}>\bar{n}_{F L}$ and $\bar{V}_{i, P L}\left(n_{P L}^{2}\right)=\bar{V}_{i, N L}>\bar{V}_{i, F L}$.

Proof: See Appendix 2.

In a first step, we focus on the main results for risk neutrality (Case 1, a)). The low membership equilibrium for PL (which is always an equilibrium; see Proposition 3 ) has lower membership and expected utility than either NL or FL, and the opposite holds for the high membership equilibrium for PL. However, we may recall that the high membership equilibrium for PL is only an equilibrium if $p \geq p^{T}$, which is very unlikely.

Karp (2012) has shown that there is no general ranking of the outcomes for NL and FL that applies to any possible set of parameter values, though it is possible to exclude the outcome where FL has both smaller expected membership and higher expected utility than NL. However, for any parameter values for which the approximation in footnote 8 holds, it would be the case that FL resulted in at least as great expected membership and at least as low expected utility as for NL (see Kolstad and Ulph 2008).

In summary, with risk neutrality, if we view the parameter values $p \geq p^{T}$ generating $n_{P L}^{2}=n_{l}$ as being rather uninteresting (i.e. we are interested in cases where the risk of high damage costs is significant), then we conclude that PL yields a lower expected coalition size and utility than either NL or FL, while for the approximation in footnote $8 \mathrm{FL}$ also yields lower 
expected utility than NL. In other words, in terms of membership, we typically expect the ranking $\mathrm{FL}>\mathrm{NL}>\mathrm{PL}$ and in terms of expected per country utility the ranking NL $>\mathrm{FL}>\mathrm{PL}$. Since the size of an agreement as such is of less importance than total welfare, the major conclusion from previous work is that learning can be bad in a strategic context of coalition formation.

In a second step, we consider what changes with risk aversion, assuming that the risk-adjusted damage cost parameter is below 1 and hence no regime shift occurs (Case 1, b) in Proposition 4). Consider first membership. From Propositions 1 and 2 we know that the size of the coalition is not affected by risk aversion under FL and decreases in $\alpha$ under NL. From Proposition 3 we know that the coalition sizes in the two possible equilibria are unaffected by risk aversion under PL. However, both the number of players and the level of risk aversion affect which equilibrium will materialize. In particular for a low number of players and high level of risk aversion, only the larger equilibrium occurs. Consequently, it follows immediately that above a threshold of risk aversion, the ranking PL $>$ NL with respect to the small PL-equilibrium $n_{P L}^{1}=n_{h}$ and hence with respect to any PL-equilibria is always true (vi); there is also a threshold above which FL $>$ NL will hold (vii). We also have PL $>$ FL if the number of players is below a threshold and the level of risk aversion is sufficiently large (viii). Thus, increasing levels of risk aversion clearly changes the ranking in terms of membership, giving PL and FL an advantage over NL.

In terms of expected utility, we know risk aversion has no impact for FL (Proposition 1), but a negative impact for NL (Proposition 2) and on each of the two equilibria for PL (Proposition 3). Consequently, above a threshold of risk aversion, this leads to the ranking FL $>$ NL because per country utility as well as membership under NL decreases with increasing risk aversion (ix). Moreover FL $>$ PL holds in terms of the smaller PL-equilibrium and may also hold for the larger PL-equilibrium above a threshold level of risk aversion (x). That is, FL improves compared to NL with increasing risk aversion continuously, and may be superior to PL above a certain threshold. The relation between PL and NL is less straightforward because both are negatively influenced by risk aversion. According to (xi), the ranking established for risk neutrality for the large PL-equilibrium, $n_{P L}^{2}=n_{l}$, namely, $\mathrm{PL}>\mathrm{NL}$, remains valid but for the small PL-equilibrium, $n_{P L}^{1}=n_{h}, \mathrm{NL}>\mathrm{PL}$ may or may not be true. Hence, overall conclusions 
are difficult, though our simulations in the next sub-section show that, on average, with increasing risk aversion PL $>$ NL eventually. Hence, the main message to take away here is that the more players are risk averse, then the more they learn, the better it is. Putting it simply, learning is good if players are risk averse.

Finally, in a third step, we address the possibility of a regime shift. Above a threshold of $\alpha$, which leads to $\hat{\gamma} \geq 1$ (case 2), NL and PL achieve the socially optimal outcome whereas this is not the case under FL. Thus, the main conclusion from step 2 above, namely that learning is good for expected utility the more risk averse players are, is now reversed once a threshold of risk aversion has been passed. In other words, learning can be bad in a strategic context of coalition formation above a threshold of risk aversion, very much like the result obtained for risk neutrality.

\subsection{Simulation Results}

In this section, we present some simulation results, based on Monte Carlo simulations, which are described in detail in Appendix III. In each simulation, first a level of risk aversion is chosen from the set $\alpha \in\{0,0.5,1,2,5,10,100,1000,10000\}$; then the number of players $N$ is generated from a discrete uniform distribution over the set $\{4, \ldots, 200\}$ and parameters $p$, $\gamma_{l}$, and $\gamma_{h}$ are generated from continuous uniform distributions over the admissible ranges, i.e. those that satisfy Assumption 2 (see sub-section 2.2). Coalition sizes and expected utilities are computed based on the expressions provide in Propositions 1-3.

In a first step, we use the Monte Carlo simulations to look at those few issues for which we could not establish analytical results. This only concerns the analysis in case 1 for which we assume $\hat{\gamma}<1$. In a second step, we present some results which compactly summarize the overall conclusion of our paper, relating it to the likelihood of case 1 and 2.

\section{$\underline{\text { Step } 1}$}

Proposition 3 showed that in case 1 risk aversion has a negative impact on expected per country utility in each of the two coalition equilibria under PL, but increases the chance of the larger equilibrium $n_{l}$ being and equilibrium, provided $p<p^{T}-$ a very likely condition, as it holds in $96.1 \%$ of the simulations. Hence, it was not clear how expected utility changes with risk aversion, assuming that in case of multiple equilibria the Pareto-superior is chosen. Table 
1, column PL, shows that on average the overall effect of increasing risk aversion on expected per country utility is negative. ${ }^{14}$

From Proposition 4, in case 1, it was not obvious how the relative ranking in terms of expected utility between PL and NL is affected by increasing risk aversion. Table 1, column $\mathrm{PL}$ and NL confirms that for risk neutrality NL $>$ PL on average. However, with increasing risk aversion, this ranking is reversed.

\section{$\underline{\text { Step } 2}$}

The analytical results distinguished between case 1 and 2. Now we ask the questions: how likely are these cases and what does this mean for the overall conclusion? Table 2 illustrates that the likelihood of case 1 constantly decreases with the level of risk aversion and the opposite is true for case 2. Hence, under PL, the average relative coalition size constantly increases with risk aversion (Table 3). This is due to two reasons. First, in case 1 the likelihood of the large equilibrium increases and the likelihood of the small equilibrium decreases. Second, the likelihood of case 2 increases compared to case 1, with the grand coalition being the outcome in case 2 with a regime shift. Under NL, the average relative coalition size first decreases and then increases (Table 3). With increasing risk aversion, Proposition 2 showed that the coalition size decreases in case 1. However, with increasing risk aversion also the likelihood of case 2 increases with the outcome that the grand coalition forms when there is a regime shift.

In terms of expected utility, a similar pattern can be observed (Table 3). It decreases under PL and NL first because the level of risk aversion has a negative impact on expected utility but this is reversed for very high levels of risk aversion because the proportion of cases in which the grand coalition forms approaches finally $100 \%$.

14 Two remarks are important regarding Table 1. First, expected utilities are negative as the maximum utility obtainable is zero in the grand coalition under all three learning scenarios. Second, in the simulations over all admissible parameter values in case 1, the increase in the average expected utility of FL for $\alpha \geq 5$ reflects the fact that the restriction $\hat{\gamma}<1$ becomes binding, i.e. parameter vectors that violate this restriction are discarded, and, as a consequence, the average coalition size, $\bar{n}_{F L}$, increases. 


\section{Summary and Conclusions}

This paper bridges two strands of literature on the formation of IEAs under uncertainty by addressing the combined roles of learning and risk aversion. This approach allowed us to explore the impact of learning for any given level of risk aversion as well as the impact of changing risk aversion under various scenarios of learning.

We generalized the model of Kolstad and Ulph (2008) who showed that with risk neutrality the possibility of learning more information about environmental damage costs generally had rather pessimistic implications for the success of the formation of IEAs. Except for a relatively small set of parameter values for which partial learning would select a high IEA membership, learning resulted in lower expected membership for partial learning and lower expected utility for both full and partial learning compared to no learning. Hence, in a strategic context, learning reduces expected utility for a wide range of parameter values. We qualified this result when players are risk averse.

Regarding the role of learning, two regimes were identified, separated by a threshold level of risk aversion. In the first regime (case 1), the level of risk aversion is below the threshold. In this regime, with increasing risk aversion, full and partial learning improve their ranking compared to no learning in terms of the size of stable coalitions and in terms of expected utility. Also full learning improves its ranking compared to partial learning in terms of expected utility. Partial and no learning are negatively affected by the direct negative impact of risk on utility. Moreover, no learning is additionally and indirectly negatively affected by a lower membership which weakly decreases with the level of risk aversion. As in Boucher and Bramoullé (2010) we found that under no learning an increase in uncertainty, in the form of a larger variance of the damage costs, or an increase in the level or risk aversion (weakly) increases signatories' abatement efforts, for a given coalition structure. Moreover, we could additionally show that an increase in the level of risk aversion (weakly) decreases the equilibrium coalition size.

In the second regime (case 2), captured in Endres and Ohl (2003) but not in Boucher and Bramoullé (2010), the level of risk aversion is larger or equal than a threshold level. In this regime, full cooperation is the equilibrium outcome under no learning and partial learning. Under no learning the reason is simply that if players are sufficiently risk averse, even without 
cooperation, they will choose the cooperative emission reduction level. Under partial learning the reason is that sufficiently risk averse players do not want to take the risk of free-riding, and therefore prefer to join the coalition. In contrast, the level of risk aversion does not affect the outcome under full learning, with an expected coalition size and utility lower than under full cooperation.

Taken together, in terms of the role of learning, the conclusions from previous papers (e.g. $\mathrm{Na}$ and Shin 1998, Kolstad 2007, and Kolstad and Ulph 2008) that learning is usually bad in a strategic context of environmental treaty formation is reversed for risk aversion below some threshold, but confirmed above this threshold. Regarding the role of risk aversion on IEA formation, we conclude that it depends crucially on the learning scenario. In particular, below a threshold level of risk aversion, coalition size decreases with increasing risk aversion under no learning (as in Boucher and Bramoullé 2010) but is likely to increase under partial learning.

In terms of the role of regime shift, the conclusion from Endres and Ohl (2003) that in a world without full information if risk aversion passes some threshold, this can have a positive impact on the success of environmental treaties was confirmed. We showed that this does not only hold for no but also partial learning and also extends to an N-country prisoners' dilemma with coalition formation. Unlike Endres and Ohl, our analysis also stressed that below the threshold, increasing levels of risk aversion may lead to worse outcomes. Under no learning, participation drops with increasing risk aversion. This is certainly an important aspect; it suggests that the climate change problem cannot simply be solved by making governments aware that they should be more risk averse in order to avoid disastrous climate change damages. As long as governments are not extremely risk averse, investment in gaining more information (i.e. learning) appears to be a more successful strategy. 


\section{References}

Arrow, K. and A. Fisher (1974), Environmental preservation, uncertainty and irreversibility. Quarterly Journal of Economics, 88: 312-319.

d'Aspremont, C., A. Jacquemin, J.J. Gabszewicz and J.A. Weymark (1983), On the stability of collusive price leadership. Canadian Journal of Economics, 16: 17-25.

Barrett, S. (1994), Self-enforcing international environmental agreements. Oxford Economic Papers, 46: 878-894.

Barrett, S. (2003), Environment and Statecraft: The Strategy of Environmental Treaty-making. Oxford University Press, New York.

Boucher, V. and Y. Bramoullé (2010), Providing global public goods under uncertainty. Journal of Public Economics, 94: 591-603.

Bramoullé, Y. and N. Treich (2009), Can uncertainty alleviate the commons problem? Journal of the European Economic Association, 7(5): 1042-1067.

Carraro, C. and D. Siniscalco (1993), Strategies for the international protection of the environment. Journal of Public Economics, 52: 309-328.

Dellink, R. and M. Finus (2012), Uncertainty and climate treaties: does ignorance pay? Resource and Energy Economics, 34: 565-584.

Endres, A. and C. Ohl (2003), International environmental cooperation with risk aversion. International Journal of Sustainable Development, 6: 378-392.

Epstein, L. (1980), Decision-making and the temporal resolution of uncertainty. International Economic Review, 21: 269-284.

Finus, M. (2001), Game Theory and International Environmental Cooperation. Edward Elgar, Cheltenham, UK.

Finus, M. (2003), Stability and design of international environmental agreements: the case of global and transboundary pollution. In: Folmer, H. and T. Tietenberg (eds.), International Yearbook of Environmental and Resource Economics 2003/4. Edward Elgar, Cheltenham, UK, ch. 3, 82-158.

Finus, M. and P. Pintassilgo (2013), The role of uncertainty and learning for the success of international climate agreements. Journal of Public Economics, 103: 29-43.

Gollier, C., B. Jullien and N. Treich (2000), Scientific progress and irreversibility: an economic interpretation of the 'Precautionary Principle'. Journal of Public Economics, 75: 229-253. 
Hong, F. and L. Karp (2013), International environmental agreements with exogenous and endogenous Risk. Mimeo.

Karp, L. (2012), The effect of learning on membership and welfare in an international environmental agreement. Climatic Change, 110: 499-505.

Kolstad, C. (1996a), Fundamental irreversibilities in stock externalities. Journal of Public Economics, 60: 221-233.

Kolstad, C. (1996b), Learning and stock effects in environmental regulations: the case of greenhouse gas emissions. Journal of Environmental Economics and Management, 31: 118.

Kolstad, C. (2007), Systematic uncertainty in self-enforcing international environmental agreements. Journal of Environmental Economics and Management, 53: 68-79.

Kolstad, C. and A. Ulph (2008), Learning and international environmental agreements. Climatic Change, 89: 125-141.

Kolstad, C. and A. Ulph (2011), Uncertainty, learning and heterogeneity in international environmental agreements. Environmental and Resource Economics, 50, 389-403 .

Markowitz, H. (1952), Portfolio selection. Journal of Finance, 7: 77-91.

Meyers, J. (1987), Two-moment decision models and expected utility maximization. American Economic Review, 77: 421-430.

Na, S.-L. and H.S. Shin (1998), International environmental agreements under uncertainty. Oxford Economic Papers, 50: 173-185.

Narain, U., A. Fisher and M. Hanemann (2007), The irreversibility effect in environmental decisionmaking. Environmental and Resource Economics, 38: 391-405.

von Neumann, J. and O. Morgenstern (1944), Theory of Games and Economic Behavior, Princeton University Press.

Rubio, S. and A. Ulph (2006), Self-enforcing international environmental agreements revisited. Oxford Economic Papers, 58: 233-263.

Saha, A. (1997), Risk preference estimation in the nonlinear mean standard deviation approach. Economic Inquiry, 35: 770-782.

Tobin, J. (1958), Liquidity preference as behavior towards risk. Review of Economic Studies, 67: 1-26.

Ulph, A. (2004), Stable international environmental agreements with a stock pollutant, uncertainty and learning. Journal of Risk and Uncertainty, 29: 53-73. 
Ulph, A. and D. Maddison (1997), Uncertainty, learning and international environmental policy coordination. Environmental and Resource Economics, 9: 451-466.

Ulph, A. and D. Ulph (1996), Who gains from learning about global warming? In: van Ierland, E. and K. Gorka (eds.), The Economics of Atmospheric Pollution. Springer, Heidelberg, ch. 3, 31-62.

Ulph, A. and D. Ulph (1997), Global warming, irreversibility and learning. Economic Journal, 107: 636-650. 


\section{Appendix I: Proof of Proposition 3}

In the second stage, the emission strategies are the same as under FL (see Proposition 1). Based on these strategies, the expected utilities, for coalition members and fringe countries, are computed using (5).

$$
\begin{aligned}
& \bar{V}_{i, P L}^{c}(n)=\left\{\begin{array}{l}
1-N \hat{\gamma} \quad \text { if } \quad n<n_{h} \\
-\hat{\gamma} N+p+(1-p) \gamma_{h} n-\alpha\left(1-\gamma_{h} n\right) \sqrt{p(1-p)} \quad \text { if } \quad n_{h} \leq n<n_{l} \\
-\hat{\gamma}(N-n) \quad \text { if } \quad n \geq n_{l}
\end{array}\right. \\
& \bar{V}_{i, P L}^{f}(n)=\left\{\begin{array}{l}
1-N \hat{\gamma} \quad \text { if } \quad n<n_{h} \\
1-\bar{\gamma} N+(1-p) \gamma_{h} n-\alpha \sqrt{p(1-p)\left[\left(\gamma_{h}-\gamma_{l}\right) N-\gamma_{h} n\right]^{2}} \quad \text { if } \quad n_{h} \leq n<n_{l} \\
1-\hat{\gamma}(N-n) \quad \text { if } \quad n \geq n_{l}
\end{array}\right.
\end{aligned}
$$

\section{First Stage}

Case 1: $0 \leq \alpha<(1-\bar{\gamma}) / \sigma\left(\gamma_{s}\right) \equiv \alpha^{T}$

(i) Applying the internal and external stability conditions in (2) and (3) in the text and using expected utilities provided in (A1) and (A2), it can be shown that there is no stable coalition with size $n<n_{h}, n_{h}<n<n_{l}$, or $n>n_{l}$. We establish in (ii) - (v) below that for all parameter values either $n_{h}$ or $n_{l}$ (or both) will be stable IEAs.

(ii) The proof is based on (iv) and (v) below, which should be consulted first. We show that for all parameter values with $0<\alpha<\alpha^{T}$ there always exists a stable IEA of size either $n_{h} \quad$ or $n_{l}$ (or both). We show first that $\tilde{\tilde{N}}>\bar{N}$. We have: $\tilde{\tilde{N}}>\breve{N} \Leftrightarrow 2 \gamma_{h}\left(n_{l}-n_{h}-1\right)>n_{l} \gamma_{l}-1$. From Assumption 2(ii) in the text (section 2.2), $n_{l}-n_{h}-1 \geq 1$ whereas $n_{l} \gamma_{l}-1<\gamma_{l}$. Hence, $2 \gamma_{h}\left(n_{l}-n_{h}-1\right)>\gamma_{h}>\gamma_{l}>n_{l} \gamma_{l}-1$.

From (iv) (a) below, $n_{h}$ is stable for all $N \geq \breve{N}$. For $N<\breve{N}<\tilde{\tilde{N}}$, we have from (iv) (b) below that $n_{h}$ is stable if $\alpha \leq \breve{\alpha}$ while from (v) (c) and (d) we have that $n_{l}$ is stable for all $\alpha$ if $p \geq p^{T}$ and for $\alpha \geq \tilde{\tilde{\alpha}}$ if $p<p^{T}$. So either $n_{h}$ or $n_{l}$ will be stable for all relevant parameter values if $\tilde{\tilde{\alpha}}<\breve{\alpha}$. Note that 
$\tilde{\tilde{\alpha}}<\breve{\alpha} \Leftrightarrow \frac{\left(p^{T}-p\right)\left(n_{l} \gamma_{l}-\gamma_{h}\right)}{\tilde{\tilde{N}}-N}<\frac{(1-p)\left(1-\gamma_{h}\right)}{\widetilde{N}-N}$ because we know that $\tilde{\tilde{N}}>\breve{N}$ and hence $\left(p^{T}-p\right)\left(n_{l} \gamma_{l}-\gamma_{h}\right)<(1-p)\left(1-\gamma_{h}\right) \Leftrightarrow\left(p^{T}-p\right)<(1-p) p^{T} \Leftrightarrow p\left(1-p^{T}\right)>0$. This completes the proof of (ii).

(iii) The results for $\alpha=0$ are shown in Kolstad and Ulph (2008).

(iv) For $\alpha>0$, it is straightforward to show that coalition size $n_{P L}^{1}=n_{h}$ is always internally stable. It is externally stable for all parameter values that satisfy:

$$
\begin{array}{r}
\alpha \sqrt{p(1-p)}\left[-N\left(\gamma_{h}-\gamma_{l}\right)-1+\gamma_{h}\left(n_{h}+1\right)+\sqrt{\left[N\left(\gamma_{h}-\gamma_{l}\right)-n_{h} \gamma_{h}\right]^{2}}\right]+ \\
(1-p)\left(\gamma_{h}-1\right)<0 .
\end{array}
$$

There are two possibilities:

(a) If $N \geq \check{\breve{N}}$ where $\check{\bar{N}} \equiv n_{h} \gamma_{h} /\left(\gamma_{h}-\gamma_{l}\right)$, then (A3) simplifies to:

$$
\left(1-\gamma_{h}\right)[(1-p)+\alpha \sqrt{p(1-p)}]>0
$$

which is true for all $\alpha>0$. If $N<\check{\bar{N}}$, then (A3) simplifies to:

$\alpha \sqrt{p(1-p)}\left[-2 N\left(\gamma_{h}-\gamma_{l}\right)+2 \gamma_{h} n_{h}-\left(1-\gamma_{h}\right)\right]+(1-p)\left(\gamma_{h}-1\right)<0$

If $N \geq \breve{N} \equiv \frac{n_{h} \gamma_{h}-0.5\left(1-\gamma_{h}\right)}{\left(\gamma_{h}-\gamma_{l}\right)}$ where $0<\breve{N}<\breve{N}$, then (A5) holds for all $\alpha>0$. So for $N \geq \breve{N}$, external stability holds for all $\alpha>0$.

(b) If $N<\breve{N}$ then (A5) holds only if

$$
0<\alpha \leq \breve{\alpha} \equiv \frac{(1-p)\left(1-\gamma_{h}\right)}{2 \sqrt{p(1-p)}\left(\gamma_{h}-\gamma_{l}\right)(\breve{N}-N)} .
$$

Using (A1) and (A2), the expected utilities are given by:

$$
\begin{aligned}
& \bar{V}_{i, P L}^{c}\left(n_{h}\right)=-\hat{\gamma} N+p+(1-p) \gamma_{h} n_{h}-\alpha\left(1-\gamma_{h} n_{h}\right) \sqrt{p(1-p)} \\
& \bar{V}_{i, P L}^{f}\left(n_{h}\right)=1-\bar{\gamma} N+(1-p) \gamma_{h} n_{h}-\alpha \sqrt{p(1-p)\left[\left(\gamma_{h}-\gamma_{l}\right) N-\gamma_{h} n_{h}\right]^{2}} \\
& \bar{V}_{i, P L}\left(n_{h}\right)=1-\bar{\gamma} N+(1-p) n_{h}\left(\gamma_{h}-\frac{1}{N}\right)-\alpha \sqrt{p(1-p)}\left[\frac{n_{h}}{N}\left(1+A-\sqrt{A^{2}}\right)+\sqrt{A^{2}}\right]
\end{aligned}
$$

where $A=N\left(\gamma_{h}-\gamma_{l}\right)-n_{h} \gamma_{h}$. 
This completes the proof of (iv).

(v) For $0<\alpha<\alpha^{T}$, it is straightforward to show that the coalition size $n_{P L}^{2}=n_{l}$ is externally stable for all parameter values. It is internally stable for all parameter values which satisfy:

$$
\begin{aligned}
& p n_{l} \gamma_{l}+(1-p) \gamma_{h}-1+ \\
& \quad \alpha \sqrt{p(1-p)}\left\{-N\left(\gamma_{h}-\gamma_{l}\right)+n_{l} \gamma_{h}-n_{l} \gamma_{l}+\sqrt{\left[N\left(\gamma_{h}-\gamma_{l}\right)-n_{l} \gamma_{h}+\gamma_{h}\right]^{2}}\right\} \geq 0
\end{aligned}
$$

Let us define $\tilde{N} \equiv \frac{\gamma_{h}\left(n_{l}-1\right)}{\left(\gamma_{h}-\gamma_{l}\right)}$ and $\tilde{\tilde{N}} \equiv \frac{n_{l}\left(\gamma_{h}-\gamma_{l}\right)+\gamma_{h}\left(n_{l}-1\right)}{2\left(\gamma_{h}-\gamma_{l}\right)}<\tilde{N}$. It is straightforward to show that $\tilde{N}>\tilde{\tilde{N}}$. Then if $N \geq \tilde{N}$ (A10) becomes:

$\left(p-p^{T}\right)\left(n_{l} \gamma_{l}-\gamma_{h}\right)-\alpha \sqrt{p(1-p)}\left(n_{l} \gamma_{l}-\gamma_{h}\right) \geq 0$

whereas if $N<\tilde{N}$ (A10) becomes:

$\left(p-p^{T}\right)\left(n_{l} \gamma_{l}-\gamma_{h}\right)+\alpha\left[2 \sqrt{p(1-p)}\left(\gamma_{h}-\gamma_{l}\right)(\tilde{\tilde{N}}-N)\right] \geq 0$

Then we have the following cases where $n_{l}$ is internally stable:

(a) $\quad N \geq \tilde{N}$; let us define

$$
\tilde{\alpha} \equiv \frac{\left(p-p^{T}\right)\left(n_{l} \gamma_{l}-\gamma_{h}\right)}{\sqrt{p(1-p)}\left(n_{l} \gamma_{l}-\gamma_{h}\right)}=\frac{p-p^{T}}{\sqrt{p(1-p)}} .
$$

Then (A10a) holds iff $p>p^{T}$ and $0<\alpha \leq \breve{\alpha}$.

(b) $\quad \tilde{\tilde{N}}<N<\tilde{N}$; let us define

$$
\tilde{\tilde{\alpha}} \equiv \frac{\left(p-p^{T}\right)\left(n_{l} \gamma_{l}-\gamma_{h}\right)}{2 \sqrt{p(1-p)}\left(\gamma_{h}-\gamma_{l}\right)(N-\tilde{\tilde{N}})} .
$$

Then (A10b) holds iff $p>p^{T}$ and $0<\alpha<\tilde{\tilde{\alpha}}$.

(c) $\quad N \leq \tilde{\tilde{N}}$ and $p \geq p^{T}$. Then (A10b) holds for $0<\alpha \leq \alpha^{T}$.

(d) $\quad N \leq \tilde{\tilde{N}}$ and $p<p^{T}$. Then (A10b) holds for $\alpha \geq \tilde{\tilde{\alpha}}$.

Using (A1) and (A2), the expected utilities are given by: 
$\bar{V}_{i, P L}^{c}\left(n_{l}\right)=-\hat{\gamma}\left(N-n_{l}\right)$;

$\bar{V}_{i, P L}^{f}\left(n_{l}\right)=1-\hat{\gamma}\left(N-n_{l}\right)$;

$\bar{V}_{i, P L}\left(n_{l}\right)=1-\hat{\gamma}\left(N-n_{l}\right)-\frac{n_{l}}{N}$.

This proves result $(\mathrm{v})$.

(vi) This is immediate from: $\frac{\partial \bar{V}_{i, P L}\left(n_{h}\right)}{\partial \alpha}=-\sqrt{p(1-p)}\left[\frac{n_{h}}{N}\left(1+A-\sqrt{A^{2}}\right)+\sqrt{A^{2}}\right]<0$.

(vii) This is immediate from: $\frac{\partial \bar{V}_{i, P L}\left(n_{l}\right)}{\partial \alpha}=-\sigma\left(\gamma_{s}\right)\left(N-n_{l}\right) \leq 0$.

Case 2: $\alpha \geq(1-\bar{\gamma}) / \sigma\left(\gamma_{s}\right) \equiv \alpha^{T}$

(viii) We argued in (i) above that for $n<n_{h}$ and $n_{h}<n<n_{l}$ there is no stable IEA. Using the external stability condition in (3) and expected utilities in (A1) and (A2), it is straightforward to show that $n_{l}$ is externally stable iff $\alpha<\alpha^{T}$. So $n_{l}$ is not a stable IEA. In (ix) below we argue that $n_{h}$ is a stable IEA and in (x) below that $N$ is a second equilibrium.

(ix) $n_{h}$ is a stable IEA under the same conditions as in (iv) above since the proof does not depend on $\alpha<\alpha^{T}$, only $0<\alpha$.

(x) It is readily shown that any possible coalition of size $n>n_{l}$ is internally stable if $\alpha \geq \alpha^{T}$; a coalition of size $N$ is externally stable by definition, so the grand coalition is always a stable IEA. However, any other possible coalition of size $n_{l}<n<N$ is only externally stable iff $\alpha<\alpha^{T}$, but we assume here $\alpha \geq \alpha^{T}$.

(xi) This follows immediately from comparing expected utilities per country, using (A1) and (A2).

\section{Appendix II: Proof of Proposition 4}

(a) $\alpha=0$

Results (i) and (ii) follow from Assumption 2(ii) and $\bar{n}_{F L}=p n_{l}+(1-p) n_{h}$.

Result (iii) is shown in Kolstad (2007). 
Result (iv) and (v) are straightforward by comparing expected payoffs per country as stated in Propositions $1-3$ for $\alpha=0$, using Assumption 2. Specifically:

$$
\begin{aligned}
& \bar{V}_{i, F L}=(1-N \bar{\gamma})+p n_{l}\left(\gamma_{l}-1 / N\right)+(1-p) n_{h}\left(\gamma_{h}-1 / N\right)(1-N \bar{\gamma})+p n_{l}\left(\gamma_{l}-1 / N\right) ; \\
& \bar{V}_{i, N L}=(N-\bar{n})(1 / N-\bar{\gamma}) ; \\
& \bar{V}_{P L}\left(n_{h}\right)=1-\bar{\gamma} N+(1-p) n_{h}\left(\gamma_{h}-1 / N\right) ; \\
& \bar{V}_{i, P L}\left(n_{l}\right)=1-\bar{\gamma}\left(N-n_{l}\right)-n_{l} / N .
\end{aligned}
$$

(b) $0<\alpha<\alpha^{T}$

Result (vi): it is readily shown by noting that $n_{N L}<n_{l}=n_{P L}^{2}$ for $\alpha=0$ and that $n_{N L}$ is weakly decreasing in $\alpha=0$ (Proposition 2, case 1). Moreover, $\hat{\gamma} \geq \gamma_{h} \Leftrightarrow \alpha \geq \sqrt{p /(1-p)} \equiv \alpha_{1}$. Hence: $\alpha<\alpha_{1} \Rightarrow n_{P L}^{1}=n_{h} \leq n_{N L} \leq n_{l}=n_{P L}^{2}$. Thus, $\alpha_{1} \leq \alpha \leq \alpha^{T} \Rightarrow n_{l}>n_{h} \geq n_{N L}$.

Result (vii): $\bar{n}_{F L}$ does not change with $\alpha ; n_{N L}=\hat{n}$ is a weakly decreasing function of $\alpha$; since $\bar{n}_{F L}>n_{h}$, there is a threshold value of $\alpha, \alpha_{2}, \alpha_{2}<\alpha_{1}$ such that $\alpha_{2} \leq \alpha \leq \alpha^{T} \Rightarrow$ $n_{N L} \leq \bar{n}_{F L}$.

Result (viii): follows from Proposition 3.

Result (ix): $\bar{V}_{i, F L}$ does not depend on $\alpha$ whereas $\bar{V}_{i, N L}$ is a strictly decreasing function of $\alpha$. For $\alpha=\alpha_{1}\left(\hat{\gamma}=\gamma_{h}\right)$ it is readily shown that:

$\bar{V}_{i, F L}-\bar{V}_{i, N L}(\hat{n})=p\left[\left(N-n_{h}\right)\left(\gamma_{h}-\gamma_{l}\right)+\left(n_{l}-n_{h}\right)\left(\gamma_{l}-1 / N\right)\right]>0$. Hence, there is a value $\alpha_{3}<\alpha_{1}$ such that $\forall \alpha, \alpha_{3} \leq \alpha \leq \alpha^{T}, \bar{V}_{i, F L} \geq \bar{V}_{i, N L}(\hat{n})$ will hold.

Result (x):

$$
\begin{aligned}
& \bar{V}_{i, F L}-\bar{V}_{i, P L}\left(n_{h}\right)=(1-p) n_{l}\left(\gamma_{l}-1 / N\right)+\alpha \sqrt{p(1-p)}\left[\sqrt{A^{2}}+\left(1+A-\sqrt{A^{2}}\right) n_{h} / N\right]>0 ; \\
& \bar{V}_{i, F L}-\bar{V}_{i, P L}\left(n_{l}\right)=-(1-p)\left(n_{l}-n_{h}\right)\left(\gamma_{h}-1 / N\right)+\left(N-n_{l}\right) \alpha \sigma\left(\gamma_{s}\right) \geq 0 \Leftrightarrow \\
& \alpha \geq\left[(1-p)\left(n_{l}-n_{h}\right)\left(\gamma_{h}-1 / N\right)\right] /\left[\left(N-n_{l}\right) \sigma\left(\gamma_{s}\right)\right] \equiv \alpha_{4} .
\end{aligned}
$$

Note that as $N-n_{l}$ decreases towards $0, \alpha_{4}$ increases, and so for some parameter values $\alpha_{4}$ will be greater than $\alpha^{T}$.

Result (xi): $\bar{V}_{i, P L}\left(n_{l}\right)>\bar{V}_{i, N L}(\hat{n}) \Leftrightarrow n_{l}>\hat{n}$, which always holds as shown in (vi). 
Result (xii): follows from (vi) and (viii) above.

Result (xiii): from Propositions 1, 2 and 3, $n_{P L}^{2}=n_{N L}=N>\bar{n}_{F L} ; \bar{V}_{i, P L}(N)=\bar{V}_{i, N L}(N)=0$; $\bar{V}_{i, F L}=p\left(N-n_{l}\right)\left(1 / N-\gamma_{l}\right)+(1-p)\left(N-n_{h}\right)\left(1 / N-\gamma_{h}\right)<0$. This completes the proof of Proposition 4.

\section{Appendix III: Description of the Monte Carlo Simulations}

i) The total number of simulations is chosen: 100,000 in all reported results.

ii) The level of risk aversion $\alpha$ is chosen, $\alpha \in\{0,0.5,1,2,5,10,100,1000,10000\}$, though results of not all values are reported here.

iii) For each simulation, the number of players $N$ is generated from a discrete uniform distribution over the set $\{4, \ldots, 200\}$.

iv) The inverse of the expected damage cost, $1 / \bar{\gamma}$, is generated from a continuous uniform distribution in the range $[3, N-1]$.

v) The inverse of the high damage cost, $1 / \gamma_{h}$, is generated from a continuous uniform distribution in the range $[2,1 / \bar{\gamma}-1]$. This ensures that $2 \leq n_{h} \leq \bar{n}-1$.

vi) The inverse of the low damage cost, $1 / \gamma_{l}$, is generated from a continuous uniform distribution in the range $[1 / \bar{\gamma}+1, N]$. This ensures that $\bar{n}+1 \leq n_{l} \leq N$.

vii) We obtain the probability of low damages from solving $p=\left(\gamma_{h}-\bar{\gamma}\right) /\left(\gamma_{h}-\gamma_{l}\right)$. and hence get: $\hat{\gamma}=\bar{\gamma}+\alpha\left(\gamma_{h}-\gamma_{l}\right) \sqrt{p(1-p)}$.

viii) This procedure only applies for the simulation reported under Step 1 in section 3.2, with restriction on $\hat{\gamma}$. If $\hat{\gamma} \geq 1 \Leftrightarrow \alpha \geq(1-\bar{\gamma}) / \sigma\left(\gamma_{s}\right) \equiv \alpha^{T}$, then go back to iii), otherwise go to ix). For the simulations reported under Step 2 in section 3.2, this procedure is redundant.

ix) The coalition sizes and expected utility follow from the equations presented in section 3. In the case of multiple equilibria under partial learning, the Pareto-superior equilibrium is chosen.

All numbers have been rounded to the third digit. Note that the number of simulations undertaken, 100,000, guarantees a low margin of error for the estimated values. For instance, for a $95 \%$ confidence interval, the maximum margin of error of an estimated probability (sample proportion) is only about 0.0016 . 